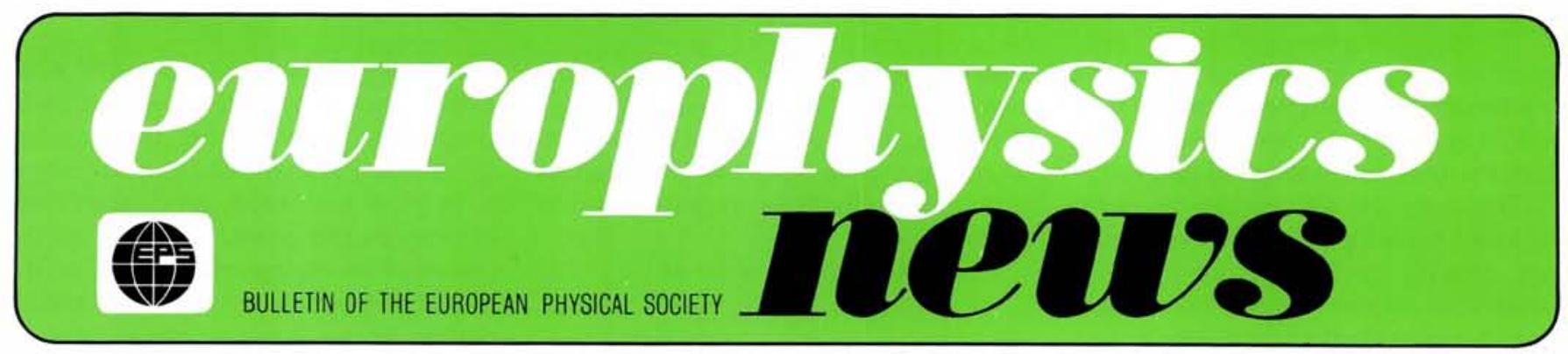

J.A.

\title{
The Cosmic Background Anisotropies
}

\section{Bianca and Francesco Melchiorri, Rome}

(Università degli Studi di Roma 'La Sapienza')

The greatest success of modern cosmology consists of having reduced the complexity of the Universe to two numbers. Even Pithagoras, the Greek philosopher who believed that everything is represented by a number, would not have hoped to achieve so much! However, a weakness remains: nobody knows the precise physical meaning of the two numbers.

The first is the ratio between the photon and baryon densities in the Universe. If we measure the radiation density, we find that in every region of space it is dominated by the $3 \mathrm{~K}$ cosmic background, which fills up every litre of space with about $10^{5}$ photons. The average density of matter is not so well known; it can be so large that it gravitationally closes the Universe, or so small that the Universe tends indefinitely to zero, if we accept a hierarchic structure for the cosmos. In the well-observed region of the Universe (a few hundred megaparsec) we believe that it is around $10^{-4}$ baryons per litre. We thus obtain the first magic number

$$
N_{1}=n_{\text {photons }} / n_{\text {baryons }} \cong 10^{9}
$$

Disregarding the exact value of this number, it is nevertheless obvious that the large excess of photons tells us something about the processes which occurred in the early Universe. George Gamow was one of the first cosmologists to point out that the large entropy of the Universe must be related in some way to the fundamental properties of matter.

Several explanations for this have been proposed, which could be classi- fied into "philosophical", astrophysical" and "sub-nuclear". The basic philosophical approach is that known as the "Anthropomorphic Principle", i.e. the photon-to-baryon ratio is so because a different ratio would render impossible the development of galaxies, stars, and finally human life on Earth. In order to have an observer, the Universe must accept the value of $N_{1}$. However meritorious this approach, one should note that the term is, in any case, quite inappropriate. As pointed out by $\mathrm{H}$. Reeves (1986) the same value of $N_{1}$ is required for "the advent on Earth of the seaurchins or the black-capped chicadees"!

The astrophysical explanation suggests that a primordial generation of stars (called Population III to distinguish them from the already existing Populations I and II) emitted photons close to the Eddington limit, which fixes the maximum luminosity for a star. These photons, eventually thermalized by interstellar matter, should constitute the bulk of the observed $3 \mathrm{~K}$ radiation, thereby determining the value of the ratio; it is important to note that the Eddington limit implies a photon/baryon ratio for a star of the same order of magnitude as the observed cosmic number.

Finally the sub-nuclear explanation observes that annihilation between matter and antimatter occurred in the beginning. The Grand Unified Theory predicts that only one baryon in every $10^{6}-10^{12}$ events survives. Therefore, one naturally expects a large excess of photons, not too different in density from the observed value.

We will not enter into a detailed discussion of the merit and demerit of each approach, since our attention will be focussed essentially on the second magic number of Cosmology.

To be more precise, it is more than a simple number. We observe in the Universe the existence of rather typical structures like stars, galaxies, clusters of galaxies, etc. How are they formed?

\section{Problem of Galaxy Formation}

The simplest idea is to suppose that they have been produced through a gravitational collapse originating from small Poissonian fluctuations in the density of matter. Unfortunately, there are no good reasons to believe that Poissonian fluc-

\section{Contents}

Cosmic Background Anisotropies 61

Fundamental Physical Constants 1986 Adjustments

International Cooperation Strengthens European Optics EPS Nuclear Winter Workshop Finite Temperature Tunnelling in Reaction Theory

Naw Individual Ordinary Members 74 2nd EPS Topical Workshop 65

New Associate Members of EPS QE Division Meeting

Physical Society of Japan, Invitation 
tuations existed in the beginning. As a matter of fact, the distance $L$ travelled by a light ray from the beginning up to a time $t$ is finite, and it tends to zero as $t \rightarrow$ 0 . Therefore, the various regions of the Universe separated by more than $L$ were not causally connected; we have no reason to expect Poissonian fluctuations for causally disconnected regions, because the Poissonian distribution requires a "thermal equilibrium". As $t$ tends to zero even the elementary particles become causally disconnected from each other. So the idea of an initial Poissonian spectrum of perturbations is completely arbitrary. If we insist on our theory and force the Universe to have Poissonian fluctuations a dilemma is created. At what time were these fluctuations present? A possible choice is Planck time before which quantum effects are dominant ( $\cong 10^{-44} \mathrm{~s}$ ), for the sole reason that, no one has been able to solve the problem up to now of how quantum effects can be included in General Relativity. Poissonian fluctuations involving the mass of a galaxy at Planck time would collapse in less than a microsecond to form black holes. This is the best reason to exclude Poissonian fluctuations at the beginning. On the other hand, if we limit the existence of Poissonian fluctuations to being inside the horizon only (i.e. inside a region causally connected) we find that a perturbation with the mass of a galaxy could enter the horizon at $t \cong 10^{8} \mathrm{~s}$. At that time a Poissonian fluctuation over $\cong$ $10^{77}$ particles (the typical number of particles in a galaxy) would be of the order of $\delta \rho / \rho=\delta N / N=\sqrt{ } N / N \cong 3 \times$ $10^{-39}$ and it has not had enough time to reach a density contrast $\delta \rho / \rho \cong 1$ by the present time. In conclusion, Poissonian perturbations at Planck time are arbitrary and produce black holes, while Poissonian fluctuations at the time when a "galaxy" is inside the horizon are too small.

This problem has been well known since the sixties, and cosmologists have found that Poissonian fluctuations should apply at $t \cong 10^{-27} \mathrm{~s}$, in order to have galaxies today. Such a time, despite all the attempts at a possible explanation cannot be associated with any peculiarities in the history of the Universe, and appears to be quite arbitrary.

In 1970, E.R. Harrison suggested for the first time that we should not be searching for the right epoch to which to apply Poissonian fluctuations, but rather selecting a suitable initial spectrum of perturbations to be applied at Planck time. Invoking a sort of democratic principle, he suggested using a scale- invariant spectrum, which provides the same power at every scale. This idea turned out to be prophetic, since a spectrum like that is naturally produced by quantum fluctuations, as pointed out by Y. Zeldovich.

Fluctuations of the order of

$$
N_{2}=\delta \rho / \rho \cong 10^{-4}
$$

are what we need to obtain galaxies today. And so we come to our second magic number, which specifies the roughness of the primordial Universe. It is now clear that it is more than a simple number, because it requires an associated perturbation spectrum and it is based not on observation (as in the case of $N_{1}$ ) but on the theory of galaxy formation through gravitational instability.

$N_{2}$ is "magic", however, in that while the spectrum can be predicted within the framework of a suitable cosmological theory like Inflation, the amplitude of the perturbations must be imposed a priori. Inflation (see Ellis J., Europhys. News 16 (1985) 9) supposes that about $10^{-32} \mathrm{~s}$ after the Big Bang, the Universe expanded exponentially, but Inflation is not able to predict the right amplitude, and a careful choice of the parameters is required in order to avoid much larger perturbations.

As far as the spectrum of perturbations is concerned, theoreticians have introduced so many symbols as to confuse everyone attempting to understand their work. Sometimes, instead of speaking of the initial spectrum at a given time (like Planck time) they prefer to discuss the spectrum when each perturbation enters the horizon. Since larger perturbations enter the horizon later, this is not a spectrum at a given time. Sometimes, instead of speaking of the spectrum in terms of density perturbations $\delta \rho / \rho$ as a function of the mass $M$ involved, they prefer to consider the local deviation $d k$ from the mean cosmic curvature $k$ produced by the density excess. As a matter of fact, the HarrisonZeldovich spectrum discussed above is characterized by $d k$ is constant and independent of $M$. The density fluctuations are, in turn, related to the curvature fluctuations $d k$ through the Field Equations of General Relativity. For example, in the case of adiabatic fluctuations (both density and radiation fluctuate) it is easy to derive the relationship

$$
\delta \rho / \rho=R(t) M^{-2 / 3} d k
$$

As pointed out before, Inflation predicts a scale-invariant spectrum of perturbations, but it is not able to anticipate the amplitude of the primordial perturbations. One might question the means used by cosmologists to reach the conclusion that perturbations must have a certain precise amplitude in order to be able to produce the galaxies. This was plotted by Lifshitz more than twenty years ago. Perturbations of small amplitude grow in an easily predictable way; in practice the growth depends on the nature of the matter involved and on the interaction between this matter and radiation. Baryonic matter has a strong interaction with radiation as long as it is ionized (Compton scattering). Therefore, it is difficult for a perturbation to grow during the first million years of the life of the Universe, unless the perturbation is much greater than the horizon, so that the photons do not have enough time to interact. At redshift $Z=1000(Z$ tends to infinity as $t$ tends to zero) it is conventionally assumed that the Universe became neutral. From that time the perturbations grew as $(1+Z)^{-1}$ up to a density contrast greater than 1 . At this stage they are strong enough to go into a non-linear regime, and the theory is too complicated to provide reliable results. If we measure the amplitude of the perturbations which are still today in the linear regime (like superclusters), we can extrapolate back these amplitudes in time at least up to $Z=1000$. By considering the processes of dissipation and damping in the radiation era we can, finally, evaluate the amplitude of the initial perturbations at Planck time. The procedure could appear cumbersome, but theoreticians are convinced that the final uncertainty is no greater than a factor five.

In conclusion, the problem of galaxy formation appears to be well defined: we have a theory which predicts a suitable spectrum of initial perturbations. Although the amplitude is not predicted by the theory, it can be estimated on empirical grounds, by extrapolating back in time the values measured in the perturbations which are still in linear regime today. Several cosmologists believe that in a not too distant future even the amplitude will be predicted by the elementary particle theories, so that the mass of a cluster of galaxies will be a parameter of nuclear physics, like the mass of a proton.

\section{The Cosmic Background Anisotropies}

The theory we have outlined above would have been universally accepted if it were not for a single fundamental problem: we are today able to observe the perturbations at $Z=1000$ by studying the spatial distribution of the cosmic background radiation, but no perturbations have been found.

There are two possible explanations for this astonishing fact:

- the interaction between the pertur- 
bations and the photons at $Z=1000$ is much weaker than expected;

- the perturbations at $Z=1000$ are much weaker than expected.

Before discussing these points let us summarize how the observations of the spatial distribution of the Cosmic Background Radiation (CBR anisotropies in the following) can provide information on the amplitude of the density fluctuations. There are three, and only three mechanisms which relate the distribution of matter to that of radiation:

1) Adiabatic fluctuations $\delta T / T=$ $K \delta \rho / \rho$. A detailed computation indicates that $K=1 / 5-1 / 30$. These fluctuations are greatest, if they exist, extending over small angular "scales" (5-10 arcminutes) where the amplitude is, however, affected by the thickness of the surface of the last scattering (perturbations much smaller than this thickness are averaged along the line of sight).

2) Random motion of matter produces anisotropies of the order of $\delta T / T \cong$ $(d / c t) \delta \rho / \rho$ where $c t$ is the dimension of the horizon. These perturbations dominate at angular scales of 1-2 degrees.

3) At very large angular scales, comparable with our horizon, the CBR photons can be affected by the gravitational potential fluctuations between us and $Z$ $=1000$. This is called the Sachs and Wolfe Effect and is of the order of $\delta T / T$ $\cong(1 / 3)(\delta \rho / \rho)(d / c t)^{2}$. These anisotropies are important at angular scales larger than a few degrees.

What we want to stress about points $(1,2,3)$ is that one is able to predict exactly the amount of the CBR anisotropies once the value of the density fluctuations at various angular scales is given.

Figure 1 shows the predictions for $\delta T / T$ in the case of what we can call the standard model, i.e. an arbitrary primordial spectrum+empirical normalization of the amplitude of $\delta \rho / \rho+$ baryonic matter. The primordial spectrum is that of Harrison-Zeldovich when $n=1$. It is clear from the comparison with observation that the already available upper limits are at variance with the predictions. The anisotropies are reduced if we increase $\Omega=\rho / \rho_{\text {crit' }}$ but values greater than 0.1 would greatly contrast with the Nucleosynthesis of primordial elements.

Before substituting the familiar baryonic matter with other kinds of matter, cosmologists have investigated the more natural and obvious possibility that CBR anisotropies are smeared out by some intervening medium between us and $Z=1000$. This is referred to as "The Secondary Ionization Problem". Any useful discussion of this problem is im-

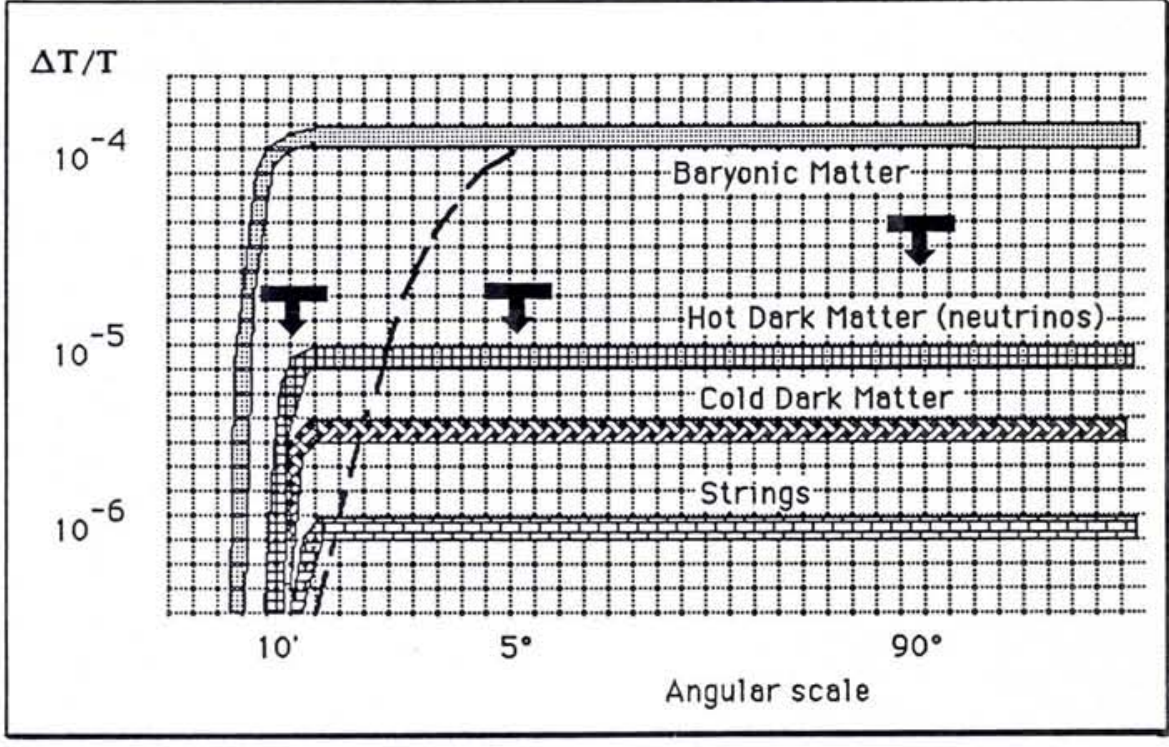

Fig. 1 - Schematic diagram of the anisotropies expected from the perturbations which have generated galaxies; the most stringent upper limits are also shown.

possible because we have too many free parameters. The main conclusions are: anisotropies over angular scales smaller than a few arcminutes are erased; anisotropies between 10 arcminutes and 1-5 degrees probably survive; anisotropies over angular scales larger than 1-5 degrees are undisturbed. This situation is qualitatively indicated by a dashed line in figure 1. The limits at angular scales larger than 1 degree exclude the classical model.

It is sad to note, however, that the anisotropies which are certainly not smeared out correspond to angular scales which are just a bit too large with respect to the structures observed today; we are not sure that the primordial spectrum of the perturbations can be extrapolated to such large scales. If, for example, there exists a sharp cut-off at scales larger than a supercluster in the spectrum of the density perturbations, and if a strong reionization occurred at $1000 \leq Z \leq 40$ our search for CBR anisotropies would be hopeless!

This situation is considered so peculiar that cosmologists have disregarded it. After all, we have no evidence for a strong early reionization and no theory exists which predicts a sharp cut-off in the spectrum of the perturbations.

Let us now discuss what can be called a "modern scenario". We introduce Inflation, so that $\Omega=1$ and the "dark matter" needed to fill up the gap between the baryonic $\Omega=0.1$ and the requirement of Inflation is presumed to be made by massive neutrinos. The fluctuations of the non-baryonic matter at $Z=1000$ can exceed those of baryonic matter because they can grow during the radia- tion era, since they are not coupled to radiation. Therefore, we may have much smaller baryonic fluctuations at $Z=$ 1000 , and we shall still be able to form galaxies through the non-baryonic fluctuations. As a consequence, the anisotropies of the CBR are reduced since they are sensitive to baryonic fluctuations only. Figure 1 shows the minimum anisotropies expected in the case of a neutrino-dominated Universe. They are marginally consistent with the upper limits.

Finally, one can speculate about other scenarios where "dark matter" is made by more exotic particles like axions or photinos. The CBR anisotropies are even smaller in this case. It is clear that these scenarios will become credible as soon as observations have ruled out the neutrino-scenario.

More recently it has been proposed that galaxies formed in a rather different way, namely, by using as cosmic seeds, topological defects called "strings" (see Europhys. News 17 (1986) 14). On average, strings would produce the smallest possible anisotropies of CBR, as shown in Figure 1. But a single string should be detectable for its peculiar spatial anisotropy: one would expect a sort of ridge in the temperature of the CBR along one side of the string. Therefore, even if the sensitivity of future observations is not able to explore the $10^{-6}$ level required by string-cosmology, one could hope to distinguish this peculiar anisotropy by its spatial signature.

Hypotheses on the origin of galaxies are certainly not exhausted in this brief review and it could well happen that some of them will produce indistin- 


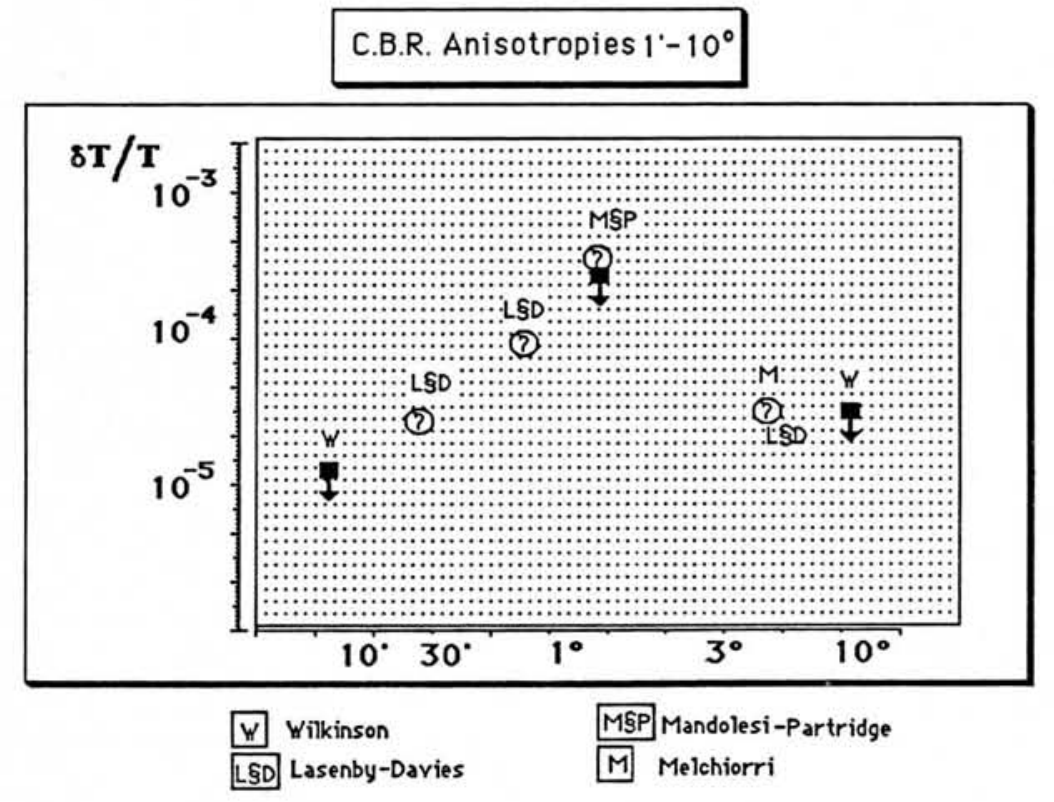

Fig. 2 - Possible detection of CBR anisotropies in the region $1^{\circ}-10^{\circ}$.

guishable signatures on CBR anisotropies. Up to now, however, a wide sample of theories proposed by cosmologists produce anisotropies different from each other in amplitude and/or in spectrum. For the observational groups working in the field, this is a rather encouraging situation. For example, a small improvement in the sensitivity already obtained would exclude or accept massive neutrinos. It would be a great success for cosmology to be able to give a definite answer on this question.

It could also happen tht the spectrum and the amplitude of the observed anisotropies are completely different from those anticipated by the theories, opening a new field of research.

\section{Possible Detections of CBR Anisotro- pies}

Before the dipole anisotropy of CBR was clearly observed and analyzed by the groups at Princeton, Berkeley and Rome-Florence, several detection claims were made, some of them indicating an amplitude and direction in good agreement with subsequent observations.

We believe it to be typical of this field of astronomy to have a range of results at the limit of the instrument sensitivity well before a new generation of detectors confirms them beyond any reasonable doubt. This was certainly true in the case of dipole anisotropy, where the observations carried out by Concklin and Henry produced figures which were considered spurious by the scientific community, but which turned out to be true in the light of present day measurements.
If we are prepared to accept this possibility, then there are good indications that CBR anisotropies have already been observed by several groups in the $1^{\circ}-10^{\circ}$ angular scale. The relevant data are plotted in Fig. 2. These are all positive detections, although none of the groups involved in this search was able to state that a systematic effect could not have produced such a result. The result of the Rome group, operating at a wavelength of $1 \mathrm{~mm}$ is at 10 standard deviations above the detector noise. This could be due to galactic dust, but any reasonable extrapolation from IRAS data excludes that.

The main reason why these results are considered as upper limits, even by the authors, is due to the fact that they lie well above the upper limit at 10 arcminutes, and are too close to the upper limit for the quadrupole. But this consideration has a bias: since the theories do not expect a bump in CBR anisotropies at angular scales of 1-5 degrees, one is led to consider such an observed bump as an upper limit. A word of caution is appropriate here. We should not forget that Concklin's results on dipole anisotropy were rejected in his time because Sandage and Tamman had found an almost zero infall velocity toward the Virgo cluster, and several cosmologists had anticipated a dipole anisotropy much smaller than $1 \mathrm{mK}$.

Speculating, one could note that such a bump, if it is not an artefact of the instruments, lies around the angular scale of the horizon at $Z=1000$. This fact could have some meaning. Perhaps the physics of matter when it enters the horizon is not yet well understood.

\section{Conclusions}

During the first half of this century cosmologists have attempted to explain physics on Earth by means of physics of the Universe. The modifications of the Einstein Field Equations proposed by Brans and Dicke were such, connecting large scale properties of the Universe with local physics through an appropriate scalar/tensor field. The dream was that of explaining, in this way, the main properties of elementary particle physics (there was even the claim of predicting the proton mass from the largescale properties of the Universe). Unfortunately the most relevant effects predicted by these theories were not observed, while others are still on the waiting list for possible future satellite experiments.

Today the situation seems to be reversed. Elementary particle physicists are on the threshold of considering a cluster of galaxies as a parameter of their theories. The Grand Unified Theory and Supersymmetry seems to be able to manage the first millimicroseconds of life of the Universe in such a way as to produce galaxies, clusters of galaxies etc. Several of the effects predicted by these theories are close to the sensitivity threshold of the instruments, and none has been clearly observed. In this context the study of CBR anisotropies plays a fundamental role: it can open a completely new physics (called by $A$. Salam "Astro-Particle Physics") or it can lead cosmologists and nuclear physicists into an impasse from which they can escape only by drastically changing their view of the role of gravity in the evolution of the Universe.

\section{REFERENCES}

The problem of primordial fluctuations within the framework of Inflation theory is discussed in: Bardeen J.M., Steinhardt P.J. and Turner M.S., Phys. Rev. D 28 (1983) 679-693.

The problem of primordial fluctuations due to strings is discussed in: de Lapparent V., Geller M.J. and Huchra J.P., Ap. J. 302 (1986) L1-5.

The computation of CBR anisotropies due to primordial density fluctuations are reported in several articles; see, for instance: Bond J.R. and Efstathiou G., A p. J. Lett. 285 (1984) L45-49.

The effect of an early reionization is discussed in: Vittorio N. and Silk J., Ap. J. Lett. 285 (1984) L39-44.

$A$ recent review of the observational data on CBR anisotropies is in: Melchiorri F., de Bernardis P., Dall'Oglio G., Masi S., Melchiorri B. and Mandolesi N., ESA Symposium on Submillimeter Astronomy, Segovia 1986. 